\title{
Artículos
}

\section{Referencia digital: concepto, tecnologías e implementación en centros de información}

\author{
Por José-Antonio Merlo-Vega
}

\begin{abstract}
Resumen: La referencia digital es el servicio que bibliotecas y centros de información ofrecen a sus usuarios para que realicen consultas a través de internet. Se describe el concepto de referencia digital y se exponen los diferentes tipos existentes a partir de diversos criterios. Se presentan las tecnologías que se emplean en la actualidad para la recepción de consultas y el envío de las respuestas. Se ofrecen las pautas para la puesta en marcha de un servicio de referencia digital partiendo de cuatro criterios: los agentes implicados, las técnicas empleadas, los recursos disponibles y la gestión del servicio. Se concluye destacando el valor de la referencia digital como un servicio que se ofrece en red y que amplía el espectro de usuarios.
\end{abstract}

Palabras clave: Referencia digital, Consultas, Servicios bibliotecarios, Servicios de información.

\section{Title: Digital reference: concept, technologies, and implementation in information centres}

Abstract: Digital reference is the service offered by libraries and information centres so that users can ask questions through the Internet. The concept of digital reference is described, along with the different typologies based on various criteria. The technologies used today for receiving questions and sending answers are presented. Guidelines are suggested for implementing a digital reference service, based on four criteria: the actors involved, the techniques used, the available resources and the management of the service. It concludes by highlighting the value of digital reference as an online service that expands the range of patrons.

Keywords: Digital reference, Reference, Libraries services, Information services.

Merlo-Vega, José-Antonio. "Referencia digital: concepto, tecnologías e implementación en centros de información". El profesional de la información, 2009, noviembre-diciembre, v. 18 , n. 6 , pp. $589-599$.

DOI: 10.3145/epi.2009.nov.02

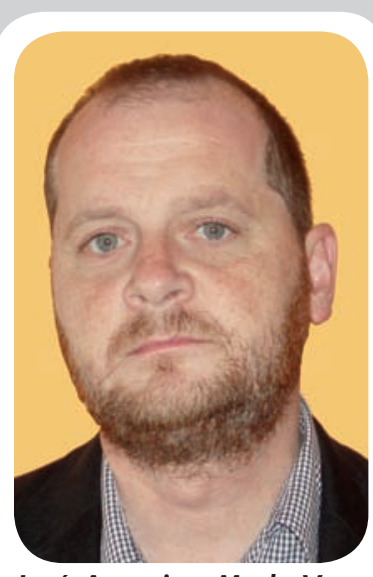

José-Antonio Merlo-Vega es doctor en documentación. Profesor titular del Departamento de biblioteconomía y documentación y director del Servicio de archivos y bibliotecas de la Universidad de Salamanca. Autor de la monografía "Información y referencia en entornos digitales: desarrollo de servicios bibliotecarios de consulta" y de diferentes artículos científicos sobre servicios de referencia y recursos de información.

\section{La referencia digital}

Los servicios que bibliotecas y centros de información ponen a disposición de los usuarios para que estos planteen consultas pueden presentarse bajo diferentes nombres, aunque es muy común el concepto de servicio de referencia.

En un sentido estricto, los servicios de referencia se deben concebir únicamente como aquéllos que dan respuesta a preguntas específicas, de información factual, que se sujetan a un sencillo código de interrogación: quién, dónde, cuándo, cómo, etc. No obstante, en muchos centros, especialmente en bibliotecas académicas y científicas, la referencia está unida a la información bibliográfica y atiende también peticiones relacionadas con búsquedas documentales y bibliográficas.

En todo caso los servicios de referencia deben identificarse con prestaciones de consulta proporcionadas por centros de información, que permiten plantear preguntas de información general sobre la biblioteca o unidad de información, además de otras factuales de respuesta rápida (pregunta-respuesta) sobre temas no relativos al centro o sus servicios y colecciones, así como de información bibliográfica, de tipo de relaciones o selecciones de documentos. Los servicios de referencia pueden definirse como las secciones de las bibliotecas y centros de información que atienden las consultas bibliográficas e informativas de los usuarios. 
Tomando como base esta concepción, en el caso de referencia digital, simplemente habrá que incluir en la definición que el proceso de consulta/respuesta se realiza por medios electrónicos. Así, la referencia digital es el servicio que proporcionan los centros documentales para atender de forma telemática demandas de información.

Entre los elementos definitorios de la referencia digital se deben destacar los siguientes:

- son servicios prestados por bibliotecas o centros de información; en definitiva, son servicios de información;

- atienden consultas, que responden a las preguntas realizadas por usuarios; internet;

- se llevan a cabo completamente en línea, desde

- están basados en tecnologías, tanto para la comunicación con los usuarios como para la resolución de las consultas.

Straw $^{1}$ estudió la relación de la referencia con los medios electrónicos. Para este autor, los referencistas han pasado "de magos a maestros", haciendo mención a la finalidad de los servicios de referencia, en sus orígenes más centrados en encontrar respuestas, pero hoy muy orientados hacia la enseñanza de fuentes de información y técnicas de búsqueda, hacia la alfabetización en información. La literatura profesional sitúa el inicio de la referencia digital en 1984, cuando se pone en funcionamiento EARS: Electronic Access to Reference Service ${ }^{2}$, de la Biblioteca de Salud de la University of Maryland at Baltimore, aunque es a lo largo de los años 90 cuando se generaliza, al expandirse internet y los centros de información comienzan a ofrecer posibilidades de comunicación telemática con los usuarios, mediante tecnologías como el correo electrónico o la misma web. Otros autores también están en sintonía con la definición de referencia digital a partir del uso de las redes. Así opina García- Delgado 3 , quien destaca la forma sobre el fondo en el proceso de la referencia digital, o Lankes ${ }^{4}$, uno de los principales investigadores en este área, quien define la voz "digital reference" en la Encyclopedia of Library and Information Science como una red de especialización, intermediación y recursos puestos a disposición de personas que buscan respuestas en entornos telemáticos. Una vez más, la definición amplía el concepto tradicional de referencia a las consultas que plantean los usuarios de forma telemática.

\section{"Los servicios de referencia digital se basan en tecnologías, tanto para la comunicación con los usuarios, como para la resolución de las consultas"}

El hecho de ser un servicio basado en internet ha provocado que se emplee de forma habitual el término "referencia virtual". El calificativo "virtual" triunfó en todas las lenguas como sinónimo de "telemático", de aquella actividad que se lleva a cabo en línea. De esta forma, se trataría de dos formas sinónimas para denominar a un mismo servicio, aunque no son las únicas, ya que es posible encontrarse con una amplia variedad de denominaciones, sobre todo si estos servicios se ofrecen también en tiempo real. Por ejemplo, en inglés es frecuente utilizar cualquiera de estos títulos: 24/7 Reference, Ask a librarian, AskA Services, Digital Reference Service, Electronic Information Service, E-Reference, Internet Information Service, Live Online Reference Service, Live Reference, Online Reference, Q\&A Services, Question Point, Virtual Reference, Virtual Reference Desk, Real Time Reference. Aunque es común el intercambio de los calificativos "virtual" y "digital", se considera más conveniente el uso del segundo. En español "virtual" representa una actividad que se asemeja a la realidad y, en el caso de los ser- 
vicios de referencia, sean presenciales o telemáticos, siempre se trata de una actividad real, con personas que se comunican y que se intercambian información. Por este motivo, en este trabajo se defiende y usa el concepto de referencia digital como el adecuado para definir los servicios de consulta que los centros de información prestan mediante internet.

\section{Tipos de servicios}

Pueden clasificarse siguiendo diferentes criterios. Según la sistematización realizada por Merlo-Vega ${ }^{5}$, se pueden agrupar bajo los siguientes elementos:

- Tipos de centros: según el tipo de unidad que presta el servicio: bibliotecas nacionales, universitarias, públicas, etc. En muchos casos, el tipo de consultas permitido está limitado en función de los objetivos del centro. Por ejemplo, es común que las bibliotecas universitarias restrinjan el servicio a información bibliográfica o que las bibliotecas nacionales sólo respondan a cuestiones sobre su ámbito geográfico o cultural. Un ejemplo de esto último es el servicio SwissInfoDesk ${ }^{6}$ de la Swiss National Library, circunscrito a consultas sobre Suiza.

- Cooperación: pueden ser individuales o cooperativos. Es frecuente que las bibliotecas que prestan este tipo de servicios pertenezcan a redes con las que comparten los recursos y la atención al usuario. Son múltiples los ejemplos de sistemas cooperativos creados para proporcionar servicios de referencia digital o que han ampliado su carta de servicios con la consulta desde internet. Destaca de forma internacional Global Reference Network ${ }^{7}$, integrada por centros de todo el mundo que emplean Question Point como sistema para administrar su referencia digital. También es un buen ejemplo la redSI@DE (Services d'information@la demande $)^{8}$, mediante la que expertos pertenecientes a bibliotecas francófonas ofrecen información especializada.

\section{"Los destinatarios del servicio determinarán dos tipos de sistemas de referencia digital, uno abierto y otro que se presta sólo a los miembros de determinada comunidad"}

- Temas: la especialización o generalidad de las respuestas también puede ser un elemento de clasificación, ya que son muchos los centros de información que no delimitan el tipo de contenidos que pueden responder, como ocurre con los servicios públicos de re- ferencia digital. Es el caso de InfoPoint ${ }^{9}$, administrado desde Alemania pero que atiende a usuarios de todo el mundo, sobre cualquier tema y en una amplia variedad de lenguas. En cambio, otros servicios están especializados en un tema, como el Agriculture Network Information Center $(\mathrm{AgNIC})^{10}$, sobre agricultura y materias afines.

- Usuarios: los destinatarios determinarán dos grandes tipos de sistemas, el que se proporciona de forma abierta y el que se presta sólo a los miembros de determinada comunidad. En el caso de bibliotecas públicas son comunes los servicios abiertos, sin restricciones o con el único límite de la lengua que se emplee, como ocurre con Pregunte: las bibliotecas responden ${ }^{11}$, donde el servicio es público siempre que las consultas se realicen en cualquiera de las lenguas oficiales del estado español. En otros casos, se emplean sistemas de identificación para que sólo los miembros de una determinada entidad lo puedan utilizar. Así ocurre en las bibliotecas universitarias, donde se suele habilitar sistemas de reconocimiento de IP, autenticación, identificación o autorización para reconocer la pertenencia del solicitante a la comunidad y darle acceso al servicio de referencia.

- Modelo del servicio: la oferta de servicios de referencia en línea no es exclusiva de los centros de información, ya que son muchos los órganos de la administración o las entidades comerciales que permiten ser interrogados por los ciudadanos para obtener informaciones concretas. Aunque sólo se pueden considerar servicios de referencia digital los que proporcionan unidades de información, existen otros modelos, como los servicios de información, los servicios de expertos o los servicios de respuestas ${ }^{12}$. Un ejemplo de servicio de información es Europe Direct ${ }^{13}$, desde el que la Comisión Europea responde a consultas sobre la Unión Europea. Los servicios de expertos pueden ejemplificarse con $\mathrm{Xpertia}^{14}$, donde personas independientes versadas en un tema responden a las preguntas recibidas en la plataforma. Por último, Yahoo Answers ${ }^{15}$ es un buen ejemplo de servicio de respuestas, donde los usuarios de internet intercambian información. Ninguno de ellos debe considerarse como referencia digital si no son ofrecidos por bibliotecas o centros de documentación.

- Tecnología: en función de las tecnologías se puede distinguir entre servicios sincrónicos y asincrónicos. La referencia sincrónica tiene lugar en tiempo real, realizándose la conversación referencista-usuario en vivo, mediante chat, mensajería instantánea, telefonía IP, videoconferencia o cualquier otro medio que permita la comunicación simultánea en linea ${ }^{16}$. Por otra parte, las tecnologías asíncronas son aquellas en las que el proceso de consulta-respuesta se realiza en diferido; es 


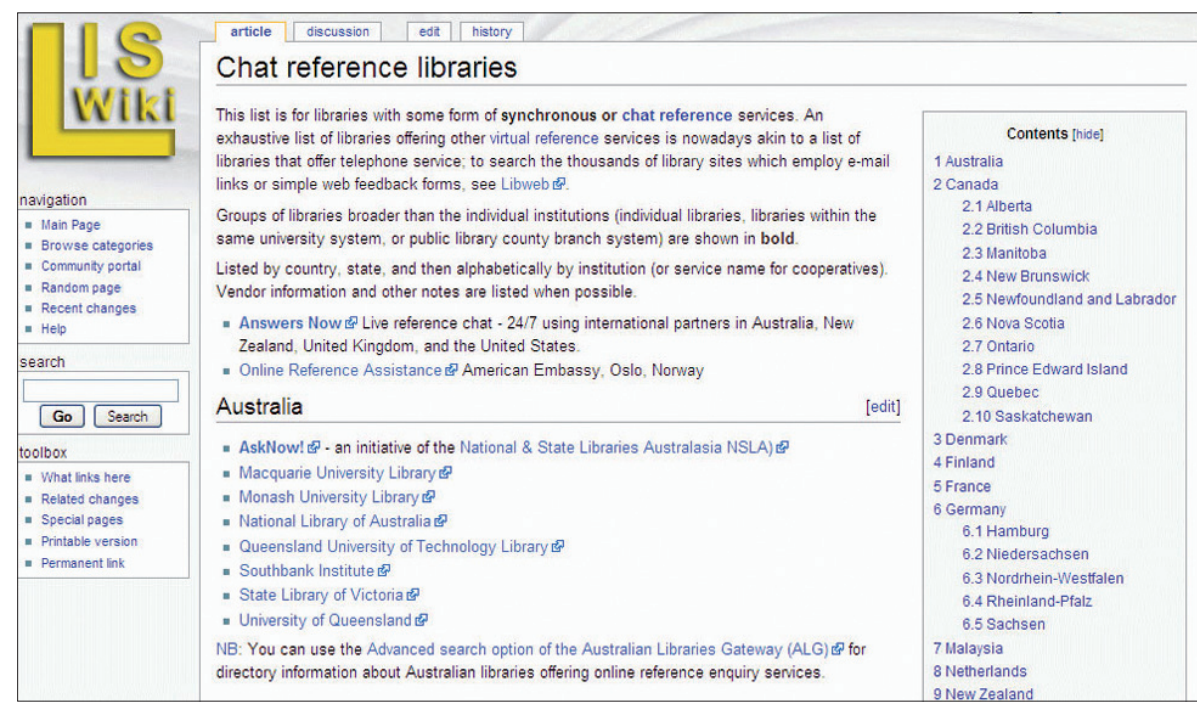

Figura 2: Liswiki recoge servicios de referencia digital de todo el mundo http://liswiki.org/wiki/Chat_reference_libraries

entre ellos los de referencia digital. En los casos en los que las bibliotecas ofrecen referencia digital para dispositivos móviles, simplemente se adaptan los contenidos web que se prestan al usuario (información, formularios, sistemas de comunicación) para que puedan ser leídos y empleados de forma correcta desde este tipo de equipamiento.

- Otros criterios: hay otros elementos que pueden servir también para clasificar la referencia digital, como la dependencia institucional, que dividiría los servicios en públicos y privados; el área geográfica, mediante la que

decir, el usuario plantea la consulta, que recibe el centro de información y que responde y envía la respuesta posteriormente. Lo habitual en este tipo de referencia digital es emplear el correo electrónico, formularios web, mensajes cortos de teléfono móvil, servicios de la web social (blogs, redes sociales) y otros medios de respuesta indirecta. No obstante, estas tecnologías (sincrónicas y asincrónicas) admiten un tercer tipo, de servicios mixtos, en los que la referencia se inicia en tiempo real y se finaliza con posterioridad, cuando el centro no puede dar la respuesta en el momento. Para ello se emplean habitualmente plataformas específicas diseñadas para la referencia digital, como Question Point, Tutor o VRL plus.

\section{"En función de las tecnologías se puede distinguir entre servicios sincrónicos y asincrónicos"}

- Dispositivos: los medios empleados para comunicarse con el centro proveedor de la referencia también permiten realizar una agrupación de servicios de consulta en línea. Así, es habitual usar ordenadores personales como el dispositivo más extendido para la referencia digital; además, se pueden encontrar servicios destinados a equipos de telefonía móvil, utilizados para la referencia mediante sms, o dispositivos inalámbricos, en los que se agruparían los medios que hacen posible la conexión a internet y que no son ordenadores; se trataría de pdas y los dispositivos tipo Ipod o PlayStation Portable, que integran servicios de telefonía, multimedia, ocio y organización de información y que permiten emplear cualquier servicio de internet, se agruparían en internacionales, nacionales, regionales y locales; o el sistema de atención, donde se podrían encontrar servicios personales o con atención humana, y automáticos, atendidos por sistemas automatizados. Hay ejemplos de todos estos subtipos de servicios de referencia digital, que en todo caso, siempre se pueden clasificar también en alguna de las categorías establecidas en los párrafos anteriores.

\section{Tecnologías}

Como se ha establecido en las páginas anteriores, la referencia digital se da siempre en línea. Los centros de información que prestan este servicio optan por soluciones tecnológicas muy diferentes para que el usuario envíe su consulta y para que el centro le envíe la respuesta precisa. Habitualmente se combinan tecnologías que hacen posible la comunicación sincrónica con aquéllas que simplemente recogen las consultas y remiten posteriormente la solución a la petición efectuada. En los siguientes párrafos se analizan las diferentes tecnologías empleadas por bibliotecas y centros de información para ofrecer servicios de referencia digital, ya sea de forma individual, empleando sólo una tecnología, o de forma mixta, utilizando varias. Para localizar ejemplos de aplicación de tecnologías, una de las fuentes más útiles es el directorio $L I S$ wiki ${ }^{17}$, donde se encuentran direcciones de servicios de referencia digital de todo el mundo, con indicaciones de los medios empleados.

La referencia digital más básica se realiza mediante correo electrónico. Aunque se trata de un sistema de comunicación común, se puede considerar un medio para la referencia digital siempre y cuando el centro informe claramente de que es válido para la consulta en línea, que los mensajes enviados a determinada dirección de correo electrónico tendrán una finalidad de 
petición de información, y de que el centro responderá por el mismo medio, mediante un mensaje de correo electrónico. Por tanto, ya sea en una web explicativa o mediante otro medio de difusión, la biblioteca o unidad de información debe indicar que el correo electrónico es la tecnología empleada para la referencia digital. Es habitual que este sistema se ofrezca junto a otros, además de que se utilize muchas veces como forma de cerrar consultas que se iniciaron en tiempo real. Se suele completar con otros servicios informativos, como envío de alertas, difusión selectiva de información, boletines de novedades, etc.

\section{"Los centros de información optan por soluciones tecnológicas muy diferentes para que el usuario envíe su consulta y para que el centro le envíe la respuesta precisa"}

Los formularios web también son un medio comúnmente empleado para la referencia digital. Los lenguajes de marca y aquéllos que se han creado para la programación web hacen posible la elaboración de sencillos formularios de recogida de los datos básicos para una consulta a la biblioteca. Cuando el usuario elige la opción de enviar, la biblioteca o centro recibe un correo electrónico con la demanda de información. No obstante, se trata de un modelo más recomendable que el simple correo electrónico, porque el centro decidirá qué información es conveniente incluir en la consulta y estructurará los datos que necesita del usuario, tanto los esenciales u obligatorios (correo y consulta, por ejemplo), como los complementarios, aquéllos que pueden contextualizar la pregunta (edad, finalidad o localidad, por ejemplo). Los formularios web permiten incluir preguntas cerradas y abiertas, lo que de alguna forma sustituye a la tradicional entrevista de la referencia presencial, es decir, la conversación que se mantiene con el usuario para delimitar la consulta. Desde el punto de vista estadístico, las demandas de información realizadas mediante formulario son fáciles de clasificar, en función de los campos que se haya decidido incluir. Puesto que un dato siempre será la dirección de correo electrónico de respuesta, se deberá extremar la seguridad para que la dirección entregada sea correcta y disponer de un espacio en la web del servicio de referencia para depositar las consultas que no han podido ser enviadas a sus destinatarios.

El chat es uno de los sistemas en tiempo real más usados. Los programas informáticos creados para la conversación en vivo en internet son muy empleados por bibliotecas y unidades de información para comunicarse con sus usuarios y atender sus peticiones de información ${ }^{18}$. Se trata de un sistema directo, que implica una serie de destrezas comunicativas, informáticas e informacionales. El uso de esta tecnología para la referencia digital requiere procedimientos precisos y bien determinados, ya que la actividad se realiza de forma simultánea, lo que implica una mayor preparación en el personal, entrevistas de referencia previamente definidas, técnicas de conversación, amplios conocimientos en recursos informativos y destreza en la recuperación de información. De igual forma, este servicio debe informar de sus horarios y de los requisitos necesarios, sobre todo si se presta de forma multimedia, aunque lo habitual es proporcionar la referencia en modo texto, mediante el intercambio de mensajes escritos con el teclado. El avance tecnológico hace posible que la aplicación de chat se inserte en la web del centro de información, de tal forma que no es necesario que el usuario disponga de un programa concreto. Algunas de las aplicaciones específicas más empleadas por las bibliotecas para la referencia digital vía chat son Live Person, PHP Live o LibraryH3lp ${ }^{19}$.

\section{"Entre las tecnologías utilizadas destacan: correo electrónico, chat, formularios web, mensajería instantánea, redes sociales, entornos virtuales, mensajería móvil, etc."}

La mensajería instantánea, muchas veces confundida con el chat, es otra tecnología muy empleada. Aunque en ambos casos la conversación se realiza de forma simultánea, estos dos servicios son tecnológicamente diferentes y emplean protocolos de comunicación distintos. No obstante, desde un punto de vista práctico, el uso es el mismo: la comunicación en tiempo real con las personas que quieran realizar consultas a la biblioteca o unidad de información. Cuando se emplea esta tecnología, se opta por dos soluciones: usar las aplicaciones de mensajería instantánea más difundidas (ICQ, Windows Live, Messenger, Google Talk, Yahoo! Messenger, Trillian, etc.) o bien utilizar widgets que integran en la web aplicaciones de mensajería instantánea (Meebome, Plugoo, Chatango, Pidgin, etc.) ${ }^{20}$. La segunda fórmula es más conveniente para los centros, ya que no es necesario que el usuario ni la unidad de información dispongan de múltiples cuentas en las diferentes aplicaciones de mensajería instantánea. Bastará con que se elija un sistema de mensajería, que la biblioteca abra una cuenta en el mismo y que inserte el 
código en su página web, de tal forma que quien lo desee se comunique con el centro mediante la ventana que encontrará en la web de la biblioteca o centro de información. Sea cual sea el sistema empleado, es necesario asegurarse de que es posible archivar las sesiones, ya que es muy útil conservar las consultas, tanto para su posterior evaluación y control estadístico, como para disponer de respuestas rápidas ante preguntas frecuentes.

Las redes sociales también se están empleando para la referencia digital. Dado que permiten la relación directa o indirecta con los usuarios o "amigos", las consultas pueden realizarse de forma inmediata, mediante los sistemas de chat integrados o bien de manera diferida, median-

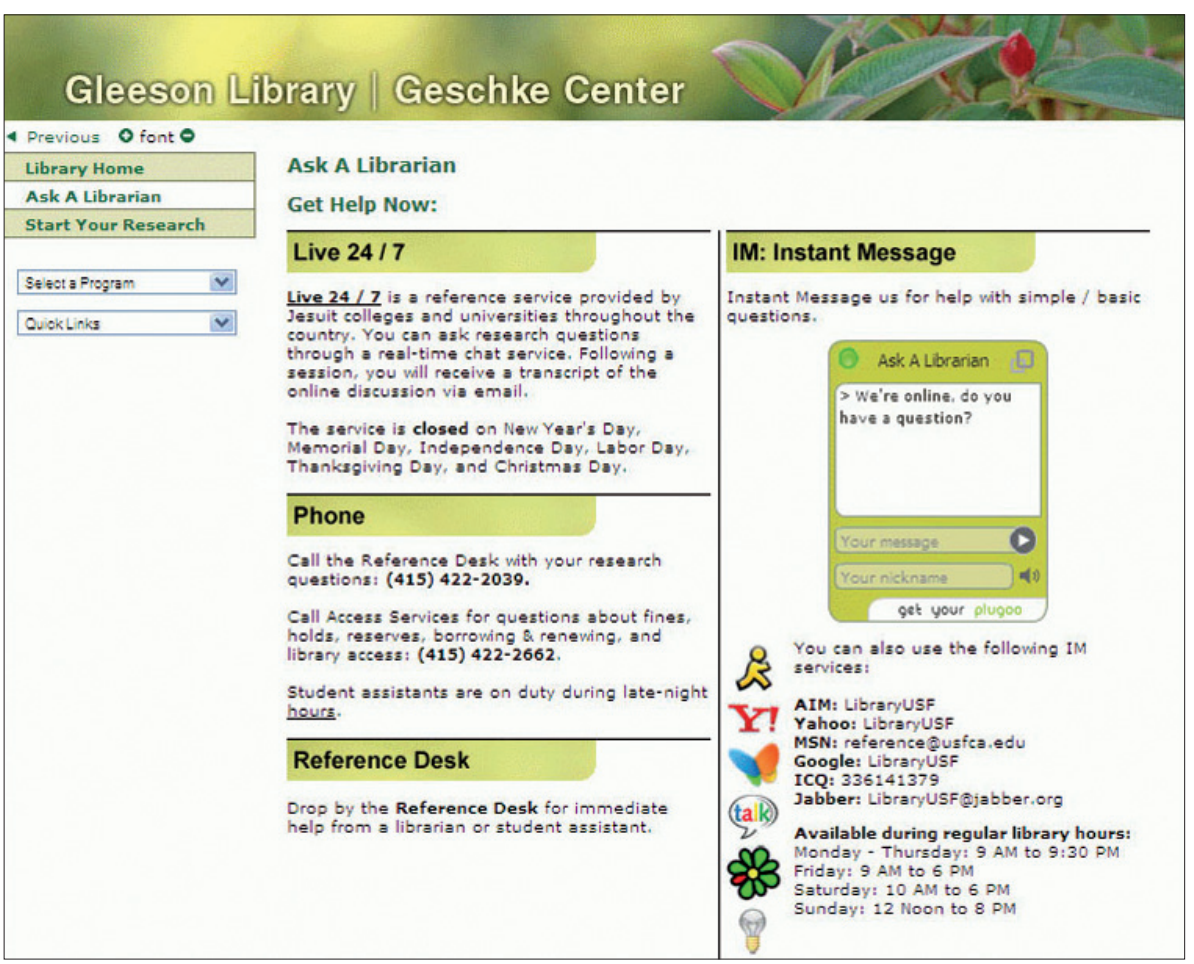

Figura 3: Los servicios de mensajería instantánea son útiles para ofrecer referencia (University of San Francisco, Gleeson Library) http://www.usfca.edu/library/ask.html

te las opciones de mensajería que proporcionan todos los servicios de redes sociales. Muchas bibliotecas están presentes en redes como Facebook, MySpace o Tuenti. La intención es prestar servicios de forma cercana, situando a las bibliotecas en los mismos entornos digitales que emplean los usuarios, para hacerles llegar informaciones y para permitir que realicen consultas. Se debe organizar este tipo de servicio, siendo constante en la presencia en la red social, realizando turnos para la atención a las posibles consultas e informando en los perfiles de la biblioteca o centro de que se presta el servicio de referencia desde la red en la que se participe. La referencia digital también se está ofreciendo en entornos virtuales, como Second life, siendo muchas las bibliotecas que disponen de simuladores de mostradores de referencia que pueden emplear los usuarios de los mundos virtuales para realizar consultas.

Los medios de comunicación telefónica se usan igualmente para la referencia digital. Así, pueden encontrarse diferentes servicios que ofrecen la posibilidad de realizar consultas vía mensajería móvil o Short Message Service (sms). Es una tecnología muy usada por los ciudadanos, por lo que las bibliotecas la pueden integrar de manera muy sencilla en su carta de servicios. Se trata de permitir consultas de tipo factual, comúnmente de carácter local, que se enviarán a un número concreto del centro informante al precio habitual de un sms. El servicio de referencia contesta por el mismo medio y habitualmente sin coste para quien ha realizado la consulta. Uno de los pioneros es Biblioteksvar ${ }^{21}$, el servicio público de referencia digital noruego, donde el usuario envía su consulta de menos de 160 caracteres al número 1980 y recibirá la respuesta en su teléfono móvil normalmente en menos de un día laborable.

En este mismo bloque hay que mencionar los servicios de referencia que se prestan mediante telefonía $I P$. Esta tecnología permite la comunicación por teclado, micrófono y cámara entre usuarios de internet y es empleada por diferentes bibliotecas como medio para recibir solicitudes de información. Para ello, se deben emplear aplicaciones específicas, entre las que destaca Skype. Como ocurre con los programas de mensajería instantánea, es necesario que el usuario disponga de la aplicación y de una cuenta, además de conocer la identificación de la biblioteca dentro del sistema de telefonía. Toda esta información se suele proporcionar desde la web del servicio de referencia. De la misma forma que con el chat, es necesario informar de los horarios en los que se encontrará en línea al personal referencista. De igual forma, hay bibliotecas que emplean éstas y otras tecnologías para la conexión mediante videoconferencia, para que el usuario plantee sus consultas, aunque en la actualidad no se trata de un servicio común.

Algunos sistemas creados para ofrecer informaciones son utilizados también para la referencia digital. Así, desde 2004 la Bibliothèque Centrale de Lyon presta su servicio Le guichet du savoir ${ }^{22}$, que está basado en las tecnologías de los foros y grupos de noticias. El usuario plantea una consulta que queda en la plataforma empleada por la biblioteca y que será contestada 


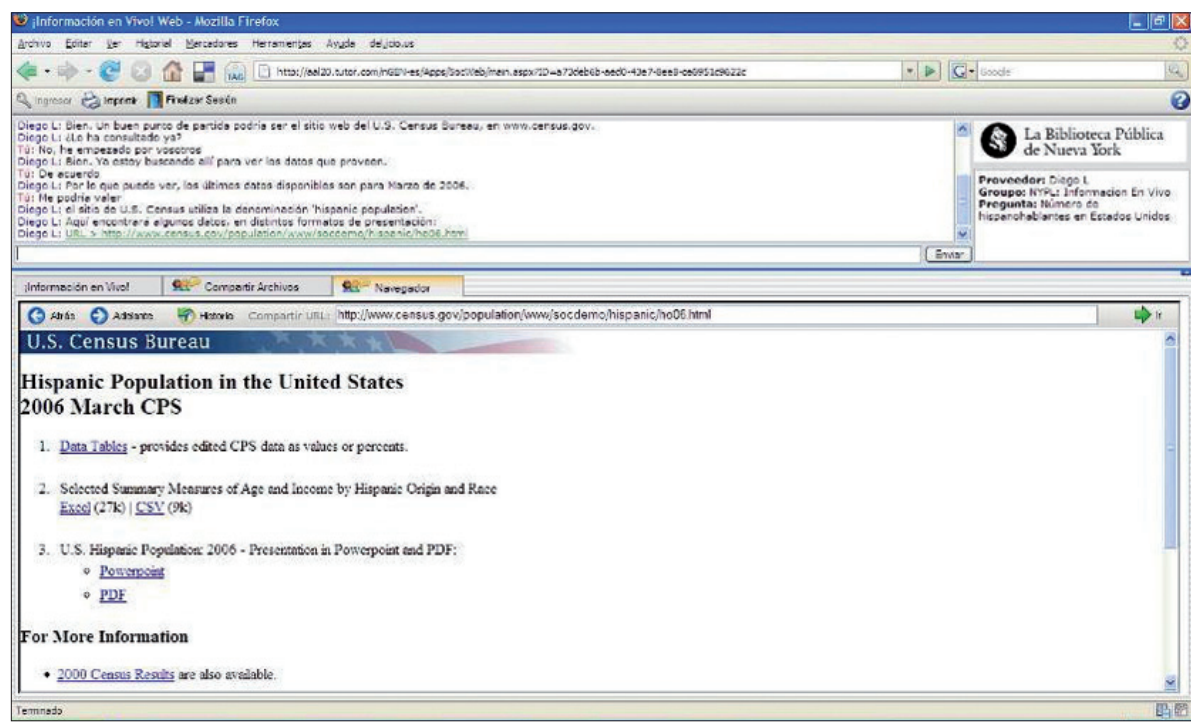

Figura 4: La conavegación simultanea la consulta en tiempo real con la presentación de páginas web

en el mismo espacio, de tal forma que tanto quien la planteó como cualquier lector, tiene a su disposición la respuesta. El archivo de preguntas y respuestas está organizado por temas y se convierte en una fuente de información con valor añadido, ya que se trata de consultas reales. Vinculado a esta tecnología por su concepción están los servicios de referencia digital que se basan en weblogs. Se trata de sistemas mixtos de atención de consultas y de publicación de información. Por una parte, la biblioteca o centro documental proporciona información mediante mensajes que publica en su blog. Por otro lado, el usuario puede emplear las opciones de comentarios para solicitar información relacionada con la publicada por el centro o bien para realizar una consulta diferente. Los blogs brindan a las bibliotecas excelentes posibilidades de publicación y redifusión de la información, así como de comunicación con sus usuarios ${ }^{23}$; no obstante, su aplicación en referencia no está demasiado extendida. Un ejemplo puede ser el blog Punto de referencia ${ }^{24}$, de la Biblioteca General de la Universidad de Puerto Rico.

La referencia digital más avanzada emplea programas cliente para la gestión de este servicio. Los centros que prestan servicios de consulta en línea, sobre todo si lo hacen de forma cooperativa, requieren aplicaciones informáticas robustas, que integren medios de comunicación, ya sea en tiempo real o de forma diferida, con el almacenamiento de las consultas y la explotación estadística de los datos. Para ello se pueden tomar diferentes decisiones, como es programar una aplicación a medida, diseñada conforme a las necesidades del centro y del servicio que quiere prestar. Otras soluciones pasan por integrar las tecnologías disponibles para la conversación (chat, mensajería, telefonía IP, etc.) o emplear programas pensados para la atención a clientes (call centers) y para servicios de información administrativa, aunque también para conferencias web y para la enseñanza en línea. No obstante la decisión más acertada es emplear programas diseñados específicamente para la referencia digital, que cumplen con los estándares bibliotecarios y que pueden ser integrados en colecciones y servicios. Se trata de soluciones comerciales, lo cual posibilita además disponer de asistencia técnica.

El mercado de este tipo de productos ha ido evolucionando, siendo el más usado Question Point (OCLC), que ofrece tanto la aplicación informática como la integración en una red mundial de centros que usan este servicio. No hay aplicaciones libres de utilidad integral para la referencia digital. Otros programas y servicios comerciales de interés son Ask a Librarian Express (Tutor. com) y VRL plus (Altarama) ${ }^{25}$. Todas estas aplicaciones se basan en la doble posibilidad de plantear la consulta mediante formularios o bien mediante comunicación instantánea.

En las solicitudes en tiempo real se debe permitir la conavegación (co-browsing). Se trata de posibilitar la comunicación en tiempo real con el usuario y ofrecer de forma simultánea sitios web en los que se encuentre la información demandada. La conavegación hace posible que el referencista y el usuario visualicen simultáneamente el mismo contenido, con la intención de que la consulta sea atendida en el momento. En todo caso, estas plataformas de referencia digital ofrecen también la posibilidad de emplear formularios web y direcciones de correo electrónico, para consultas en diferido o para finalizar sesiones en las que la información demandada no haya podido ser entregada en tiempo real. De igual forma, lo idóneo es que estos programas cumplan la norma Question/Answer Transaction Protocol ${ }^{26}$, aprobada por NISO en diciembre de 2006 y que sirve como base a las plataformas de referencia digital que integran información de los usuarios, recursos de información, archivo de preguntas y respuestas, que están destinados al trabajo cooperativo y que deben permitir la interoperabilidad de sistemas.

\section{Implementación de servicios de referencia digital}

A pesar de tratarse de servicios recientes, hay suficientes directrices que pueden ser tomadas en cuenta a la hora de planificar este tipo de servicio. La IFLA aprobó en 2003 las Recomendaciones para el servicio de referencia digital ${ }^{27}$. En estas pautas se delimita el 


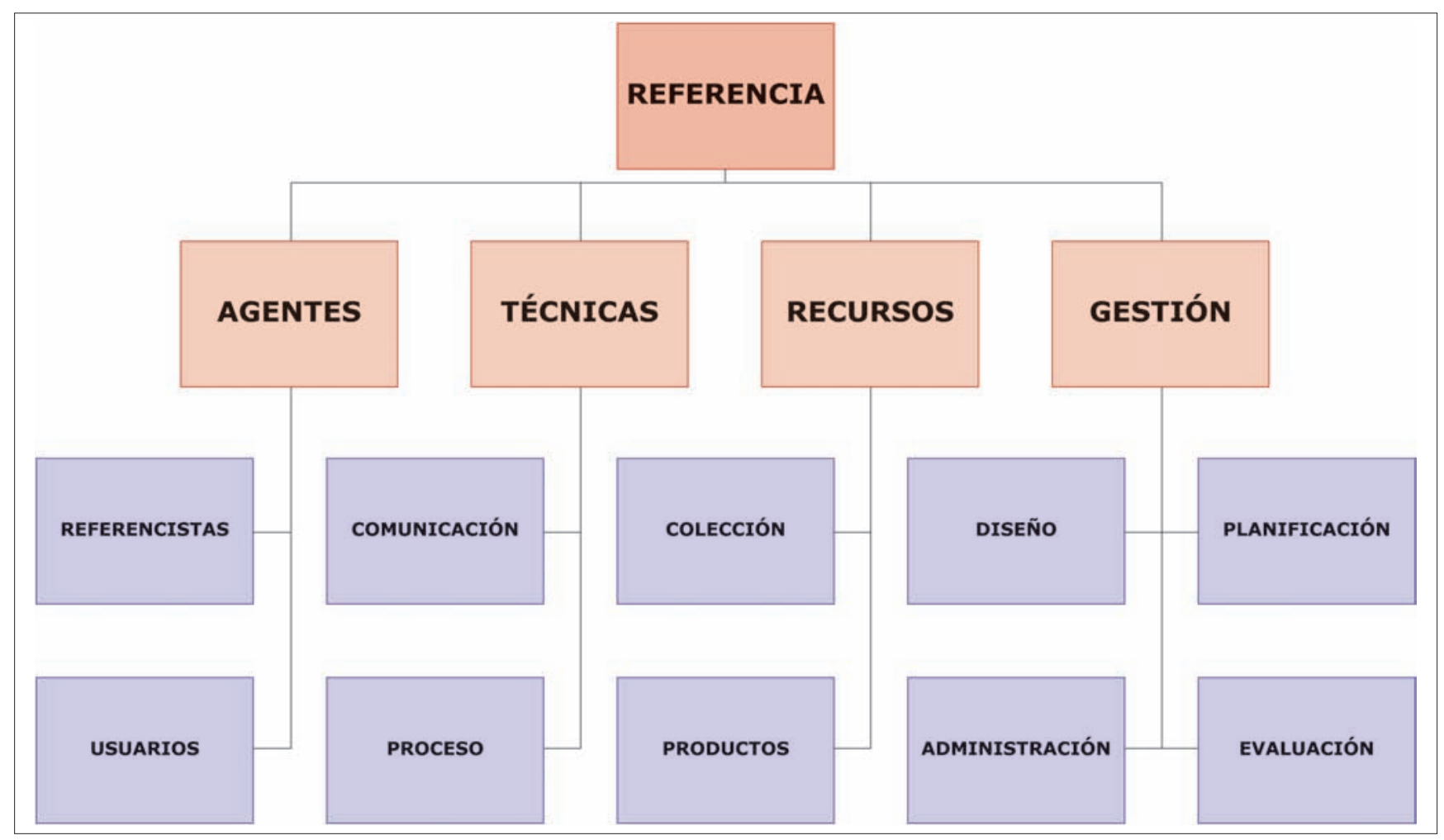

Figura 5: Elementos que componen el servicio de referencia digital

concepto de referencia digital, ofreciendo la contextualización de dichas directrices, que quieren contribuir tanto a la administración de este tipo de servicios, como a la normalización de la práctica de los mismos. Se trata de recomendaciones especialmente concebidas para servicios cooperativos, tanto en su prestación conjunta como en el uso común de recursos. El primer bloque de estas pautas de la IFLA trata sobre la gestión de la referencia digital, aportando recomendaciones sobre la política de referencia, la planificación del servicio, el personal que lo atenderá y su formación, la interfaz del servicio y aspectos de tipo legal, de difusión, calidad y cooperación. El segundo gran bloque está centrando en la práctica, proporcionando consejos generales para la prestación y recomendaciones específicas sobre el contenido del servicio o sobre cómo debe realizarse si se emplean aplicaciones de chat.

Por otra parte, la Reference and User Services Association (RUSA), vinculada a la American Library Association ( $A L A)$, ha publicado diferentes pautas relacionadas con la referencia en entornos digitales. Entre ellas destacan sus Guidelines for implementing and maintaining virtual reference services ${ }^{28}$, de 2004. Como en el caso de las pautas de la IFLA, las de la RUSA proporcionan una definición de referencia digital, incluyen consejos generales para la preparación de este tipo de servicios, así como indicaciones particulares sobre usuarios, procedimientos, organización del servicio, presupuestos, personal, evaluación, etc. Además de las directrices de la IFLA y la RUSA, de- ben mencionarse las que en su momento difundió la desaparecida Virtual Reference Desk Network bajo el título de Facets of quality digital reference services ${ }^{29}$, que han servido de base para la puesta en práctica de un elevado número de servicios de referencia digital, sobre todo en Estados Unidos.

\section{"La referencia digital multiplica el alcance de los servicios de información"}

Las directrices de IFLA y RUSA, junto a normas recogidas en manuales de procedimiento de diferentes servicios bibliotecarios de consulta desde internet y a las impresiones de los autores que han investigado sobre planificación y evaluación de la referencia digital, pueden agruparse a partir de los componentes que intervienen en la referencia digital: agentes, técnicas, recursos y gestión. Cada uno de estos grupos puede subdividirse asimismo, como se muestra en la figura 5, para ofrecer algunas pautas que hay que tener en cuenta para implementar un servicio de este tipo ${ }^{30}$.

El primer grupo de recomendaciones se refiere a los "agentes", integrados por el personal referencista y los usuarios. El perfil de los referencistas requiere una formación profesional sólida y en continuo reciclaje. Deberán poseer competencias específicas en tres cam- 
pos: comunicación personal, aspectos tecnológicos y destrezas informacionales. La biblioteca o unidad de información deberá establecer a qué usuarios dirige su servicio de referencia digital y, en el caso de restringir el acceso, emplear sistemas de autenticación y autorización.

El segundo elemento que integra el sistema de la referencia digital son las "técnicas", en las que se incluyen los sistemas mediante los que se establece la comunicación con los usuarios y el proceso que se sigue para la resolución de las consultas. En los sistemas de comunicación se debe optar por un método de recepción de consultas y envío de respuestas que tenga en cuenta la disponibilidad del personal de la biblioteca. No se puede elegir un sistema en tiempo real si no se dispone del volumen de referencistas necesarios. Si es posible, se deben emplear aplicaciones que permitan la conavegación, ya que se trata de la herramienta más útil para mostrar en tiempo real la información demandada, consiguiendo resultados satisfactorios en el instante. Sobre el proceso de referencia, la primera indicación debe hacerse sobre la actitud, que debe ser amable, de servicio público, siempre demostrando predisposición para informar. Es importante transmitir que se está siendo atendido por profesionales de la información. Será necesario marcar una duración en las consultas en tiempo real, para no saturar el servicio. Para conseguir mayor agilidad, se debe disponer de un archivo de mensajes pre-escritos, que puedan ser usados ante situaciones o consultas similares. También es recomendable informar al usuario en todo momento de la actividad que se está realizando: "estoy buscando", "estoy comprobando", "le envío el documento", etc.

\section{"Los servicios de referencia pueden traspasar su misión como puntos de información para posicionarse como proveedores de datos o documentos y como asesores en información"}

Los "recursos" de información forman el tercer apartado de elementos en la cadena de la referencia digital. La colección de referencia comienza por el fondo físico, aunque en referencia digital gran parte de las fuentes de información están accesibles desde internet. Es necesario que los referencistas dispongan de amplios conocimientos en recuperación de información, de dónde y cómo se puede conseguir un dato o un documento. Además de las colecciones propias y ajenas, los responsables de la referencia digital deben elaborar productos informativos, como selecciones de recursos,

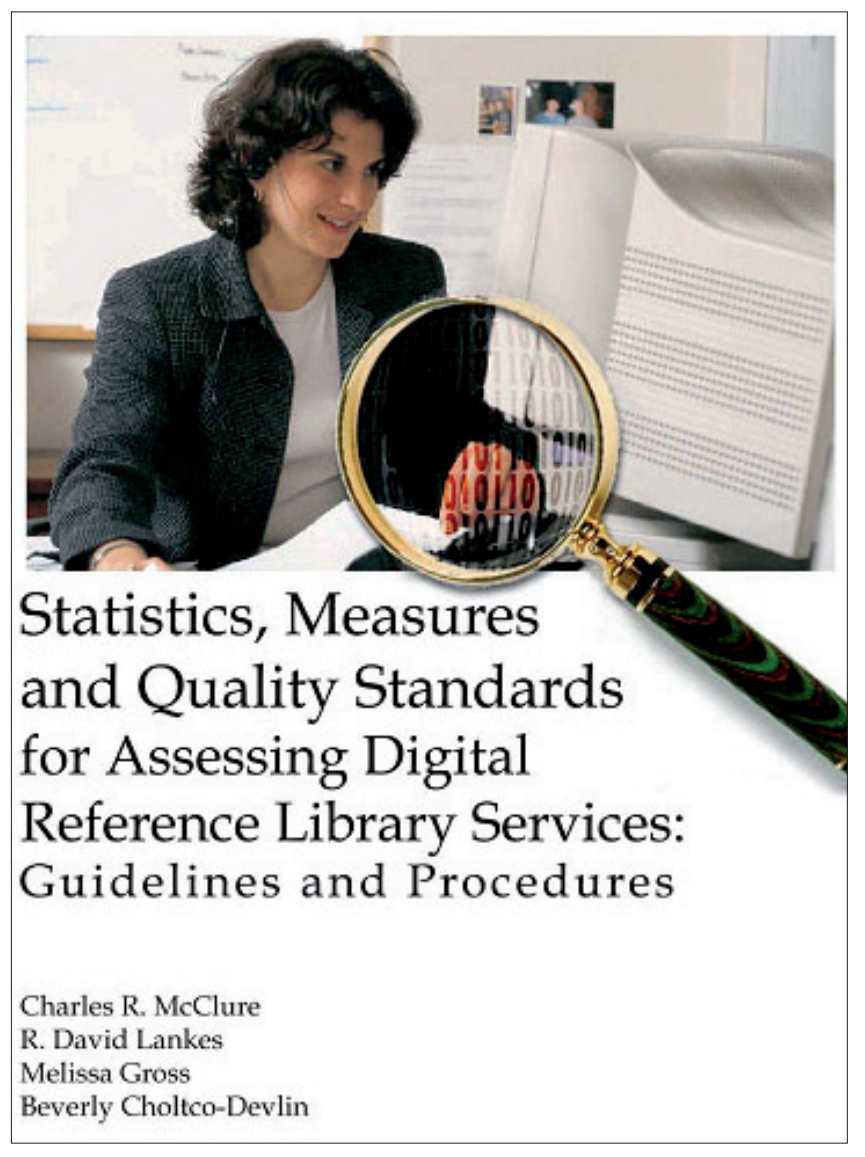

Figura 6: Estadísticas, medidas y normas de calidad para la evaluación de servicios bibliotecarios de referencia http://quartz.syr.edu/quality/Quality.pdf

guías informativas, tutoriales, boletines, alertas, etc., que en muchos casos solucionarán las posibles consultas de los usuarios. Además, el archivo de respuestas ofrecidas por el servicio es una buena base de datos de conocimientos, por lo que sus contenidos pueden difundirse en el sitio web del servicio de referencia digital.

El último grupo de elementos integrantes de la referencia digital está formado por los relativos a la "gestión": diseño, planificación, administración y evaluación. En el diseño se deben contemplar tanto las particularidades espaciales y de equipamiento del lugar físico desde donde se atiende la referencia, como la consistencia, seguridad y rapidez de las redes de telecomunicaciones sobre las que se prestará el servicio. La planificación de la referencia digital debe plantearse de forma sostenible, siempre a medio y largo plazo, aunque es útil comenzar con proyectos piloto de menor duración. La filosofía de un plan para referencia digital tiene que ser coherente con la política general del centro y la entidad, estableciendo un modelo específico de servicio y redactando manuales de procedimiento para todas las áreas de trabajo, en los que se definan los tipos de información que se facilitarán (básica sobre la biblioteca o centro, orientación e instrucción sobre servicios y recursos, información factual general o específica, investigación bibliográfica y documental), así 
como los modelos de respuesta y la política de suministro de documentos. Además, se debe tener clara la administración del servicio, que debe estructurarse en un organigrama en el que se definan jerarquía, responsabilidades, funciones y tareas. La organización debe tener en cuenta un sistema de turnos y de asignación de consultas, los horarios de atención y los plazos de entrega de respuestas, que deben ser marcados a partir de las posibilidades reales del centro que presta la referencia digital. Por último, hay que tener presente que es necesario un sistema de evaluación, por lo que debe decidirse de antemano qué datos se recogerán para su posterior análisis. Es recomendable analizar el funcionamiento del servicio, las capacidades de los profesionales, la utilidad de los recursos empleados para la referencia y la calidad de las informaciones facilitadas. Para la evaluación de este tipo de servicios, existen un documento muy valioso, dirigido por McClure ${ }^{31}$, en el que se presentan treinta y cinco medidas, estadísticas y estándares perfectamente comentados y con los datos necesarios para tomar dichas medidas.

\section{Dos ideas conclusivas}

La referencia digital multiplica el alcance de los servicios de información. En una sociedad donde la presencia física no es necesaria para la realización de muchas tareas, las bibliotecas y unidades de información deben aprovechar las tecnologías para poner en valor su bagaje como centros especialistas en información. Los servicios de referencia pueden traspasar su misión como puntos de información para posicionarse como proveedores de datos o documentos y como asesores en información.

La particularidad esencial de la referencia digital es que se trata de un servicio que se ofrece en línea. No obstante, el mero hecho de usar tecnologías para recibir consultas de los usuarios no implica la correcta prestación del servicio. Es preciso delimitar el modelo, establecer objetivos, disponer de manuales de procedimientos y controlar de forma precisa el sistema de administración, incluyendo presupuestos, personal y recursos y teniendo en cuenta la necesidad de evaluar el servicio, tanto los aspectos cuantitativos (número de preguntas, por ejemplo), como la calidad del mismo (satisfacción del usuario, pertinencia de las respuestas, etc).

\section{Notas}

1. Straw, Joseph E. "From magicians to teachers: The development of electronic reference in libraries: 1930-2000". The reference librarian, 2001, n. 74 , pp. 1-12.

2. Weise, Frieda 0.; Borgendale, Marilyn. "EARS: Electronic Access to Reference Service". Bulletin of the Medical Library Association, 1986, v. 74, n. 4, pp. 300-304.

3. García-Delgado, Purificación. "El servicio de referencia digital". El profesional de la información, 2003, v. 12, n. 4, pp. 320-330.
4. Lankes, R. David. "Digital reference". En: Drake, Miriam (ed.). Encyclopedia of library and information science. New York: Marcel Dekker, 2003.

5. Merlo-Vega, José-Antonio. "Tipología de los servicios de referencia digital”. En: Merlo-Vega, José-Antonio. Información y referencia en entornos digitales: desarrollo de servicios bibliotecarios de consulta. Murcia: EDITUM, 2009, capítulo 7.2.

6. SwissInfoDesk

http://www.nb.admin.ch/dienstleistungen/swissinfodesk/index.html

7. Global Reference Network

http://www.loc.gov/rr/digiref

8.SI@DE (Services d'information @la demande)

http://www.bnf.fr/PAGES/accedocu/siade.htm

9. InfoPoint

http://www.zlb.de/fragen_sie_uns/ask_a_librarian

10. Agriculture Network Information Center (AgNIC)

http://www.agnic.org

11. Pregunte: las bibliotecas responden

http://www.pregunte.es

12. Los diferentes modelos se pueden consultar en: Merlo-Vega, José-Antonio. "Referencia digital y servicios electrónicos de información". Anuario ThinkEPI 2009, EPI SCP, pp 137-140.

13. Europe Direct

http://ec.europa.eu/europedirect

14. Xpertia

http://www.xpertia.com

15. Yahoo Respuestas

http://es.answers.yahoo.com

16. Marsteller y Schmitt-Marsteller analizaron las tecnologías sincrónicas de mayor efectividad hasta ese momento para la referencia digital en bibliotecas. Véase: Marsteller, Matthew R.; Schmitt-Marsteller, Jackie. "Opportunities for real-time digital reference service". The reference librarian, 2002, v. 38, n. 79-80, pp. 163-181.

17. LIS wiki: Chat reference libraries http://liswiki.org/wiki/Chat_reference_libraries

18. Entre los múltiples trabajos realizados sobre el chat como medio para la referencia destaca: Francoeur, Stephen. "An analytical survey of chat reference services". Reference services review, 2001, v. 29, n. 3, pp. 189-204.

19. Live Person

http://solutions.liveperson.com

PHP LiveL

http://www.phplivesupport.com

Live H3lp

https://admin.libraryh3lp.com

20. Desai ha estudiado en varias ocasiones las aplicaciones de mensajería instantánea que son de utilidad para la referencia digital. Véase, por ejemplo: Desai, Christina M. "Instant messaging reference: how does it compare?”. The electronic library, 2003, v. 21, n. 1, pp. 21-30.

21. Biblioteksvar

http://biblioteksvarno

22. Le guichet du savoir

http://www.guichetdusavoir.org

23. Las posibilidades de los blogs en referencia digital han sido estudiadas en: Pomerantz, Jeffrey; Stutzman, Frederic. "Collaborative reference work in the blogosphere". Reference services review, 2006, v. 34, n. 2, pp. 200-212.

24. Punto de referencia

http://blogs.uprm.edu/puntodereferencia

25. Question Point

http://www.questionpoint.org

Ask a Librarian Express

http://www.tutor.com/libraries/virtual-reference 
VRL plus

http://www.altarama.com/page/VRLplus.aspx

26. NISO. Networked reference services: Question/Answers Transaction Protocol. Bethesda: NISO Press, 2006.

http://www.niso.org/publications/tr/TR04-NetRefQAProtocol.pdf

27. IFLA, Reference and information services section. Recomendaciones para el servicio de referencia digital. The Hague: IFLA, 2004.

http://archive.ifla.org/VII/s36/pubs/drg03-s.htm

28. RUSA. Guidelines for implementing and maintaining virtual reference services. Chicago: ALA, RUSA, 2004.

http://www.pla.org/ala/mgrps/divs/rusa/resources/guidelines/virtrefguidelines.cfm

29. La versión quinta y definitiva de Facets of quality for digital reference services, correspondiente a junio de 2003.

http://www.webjunction.org/quality-standards/articles/content/438969

30. Los siguientes párrafos proporcionan un resumen de la guía de cincuenta aspectos que hay que contemplar para llevar a la práctica un servicio de referencia digital y que se recogen en:

Merlo-Vega, José-Antonio. "Planificación y creación de un servicio de referencia digital”. En: Merlo-Vega, José-Antonio. Información y referencia en entornos digitales: desarrollo de servicios bibliotecarios de consulta Murcia: EDITUM, 2009, capítulo 8.1.

31. McClure, Charles R.; Lankes, R. David; Gross, Melissa; CholtcoDevlin, Beverly. Statistics, Measures and Quality Standards for Assessing Digital Reference Library Services: Guidelines and Procedures. Syracuse, New York; Tallahassee, Florida: Information Institute of Syracuse; School of Information Studies, 2002.

http://quartz.syr.edu/quality/Quality.pdf

\section{Bibliografía}

Anderson, Byron; Webb, Paul T. (eds.). New directions in reference. Binghamton, NY: The Haworth Information Press, 2006.

Bridgewater, Rachel; Cole, Meryl B. Instant messaging reference: a practical guide. Oxford: Chandos, 2009.

Cassell, Kay Ann; Hiremath, Uma. Reference and information services in the 21st century: an introduction. 2nd ed. London: Facet, 2009.

Coffman, Steve. Going live: Starting and running a virtual reference service. Chicago: American Library Association, 2003.

Guo, Jing; Pan, Wei; Zheng, Qiaoying; Huang, Min; Yang, Zongying; Ye, Ying. "Design an ideal Digital Reference Service (DRS): model for academic libraries". En: Digital Libraries: International Collaboration and Cross-Fertilization. Berlin: Springer, 2004, pp. 668-669.

Janes, Joseph. Introduction to reference work in the digital age. New York: Neal-Schuman, 2003.

Katz, William A. (ed.). Digital Reference Services. Binghamton, NY: The Haworth Information Press, 2004.

Kern, M. Kathleen. Virtual reference best practices: tailoring services to your library. Chicago: ALA, 2009.

Kimmel, Stacey; Heise, Jennifer (eds.). Virtual reference services: issues and trends. Binghamton, NY: The Haworth Information Press, 2003.

Kirko, Buff; Ross, Mary Bucher. Virtual reference training: the complete guide to providing anytime, anywhere answers. Chicago: American Library Association, 2004
Kovacs, Diane K. The virtual reference handbook: interview and information delivery techniques for the chat and e-mail environments. London: Facet, 2007.

Lankes, R. David; Abels, Eileen; White, Marilyn; Haque, Saira N. (eds.). The virtual reference desk: creating a reference future. London: Facet, 2006.

Lankes, R. David; Collins, John W.; Kasowitz, Abby S. (eds.). Digital reference service in the new millennium: planning, management, and evaluation. New York, London: Neal-Schuman, 2000.

Lankes, R. David; Janes, Joseph; Smith, Linda C.; Finneran, Christina M. (eds.). The virtual reference experience: integrating theory into practice. New York: Neal-Schuman, 2004.

Lankes, R. David; Kasowitz, Abby S. The AskA starter kit: how to build and maintain digital reference services. Syracuse, New York: Syracuse University, 1998.

Lankes, R. David; McClure, Charles R.; Gross, Melissa; Pomerantz, Jeffrey (eds.). Implementing digital reference services: setting standards and making it real. New York: Neal-Schuman, 2002.

Lankes, R. David; Nicholson, Scott; Radford, Marie L.; Silverstein, Joanne L.; Westbrook, Lynn; Nast, Philip (eds.). Virtual reference service: from competencies to assessment. New York: Neal-Schuman, 2007.

Lipow, Anne-Grodzins. The virtual reference librarian's handbook. New York: Neal-Schuman, 2003.

Manso-Rodríguez, Ramón-Alberto. Servicio de referencia virtual: concepto, organización y evaluación. Santa Clara: Editorial Feijóo, 2006.

McMenemy, David; Poulter, Alan. Delivering digital services: a handbook for public libraries and learning centres. London: Facet, 2005.

Meola, Marc; Stormont, Sam. Starting and operating live virtual reference services: a how-to-do-it manual for librarians. New York: Neal-Schuman, 2002.

Merlo-Vega, José-Antonio. Información y referencia en entornos digitales: desarrollo de servicios bibliotecarios de consulta. Murcia: EDITUM, 2009.

Pantry, Sheila. Creating a successful e-information service. London: Facet, 2002

Rodríguez-Briz, Fernanda. Los servicios de referencia virtual: surgimiento, desarrollo y perspectivas a futuro. Buenos Aires: Alfagrama, 2005.

Ronan, Jana Smith. Chat reference: a guide to live virtual reference services. Westport, Conn.: Libraries Unlimited, 2003.

Steiner, Sarah K.; Madden, M. Leslie (dirs.). The desk and beyond: Next generation reference services. Chicago: Association of College and Research Libraries, 2008.

West, Jessamyn (ed.). Digital versus non-digital reference: ask a librarian online and offline. Binghamton, NY: The Haworth Information Press, 2004

Wood, M. Sandra. Medical librarian 2.0: use of web 2.0 technologies in reference services. Binghamton: The Haworth Press, 2007.

José-Antonio Merlo-Vega. Universidad de Salamanca. merlo@usal.es

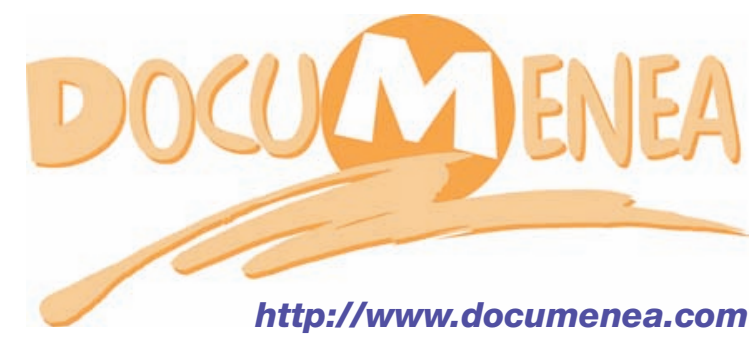

En DoculMenea sabemos casi todo lo que ocurre en biblioteconomía, documentación, comunicación y nuevas tecnologías de la información. seleccionamos lo que hay que saber: 


\section{Rapidez, precisión, flexibilidad}

En el siglo XXI, más que nunca, la información es poder. Ayudar a empresas e instituciones a gestionar y rentabilizar su caudal informativo, tanto el que poseen como el que genera diariamente su actividad, es el objetivo y la razón de ser de Baratz.

www.baratz.es 